\section{Health effects of the CPT1A P479L variant: responsible public health policy}

To the Editor: In "Carnitine Palmitoyltransferase 1A P479L and Infant Death: Policy Implications of Emerging Data," Fohner et al. ${ }^{1}$ present a reanalysis and discussion of published data regarding the health effects of a single-nucleotide variant $($ c.1436C $\rightarrow$ T; p.P479L) in the carnitine palmitoyltransferase 1A (CPT1A) gene that has a high prevalence among indigenous populations from western and northern Alaska, Vancouver Island, and northern coastal regions of Canada, Greenland, and northeast Siberia. They include an assessment of whether an association between the variant and infant mortality that has been reported in studies from Canada ${ }^{2,3}$ and the United States ${ }^{4,5}$ fulfills epidemiological criteria for establishing a causal association. Their conclusion is that the evidence is insufficient to support a causal relationship, and they recommend further research. They also suggest that published evidence of deleterious health effects associated with homozygosity for the variant is insufficient to warrant public health interventions.

The authors' statements regarding causation reflect a misunderstanding of causation assessment in epidemiological studies, as do their statements regarding the level of certainty necessary for public health action. Observational epidemiological studies are not designed to assess causation but to determine associations. Nevertheless, such studies can provide powerful justification for public health interventions. Among hundreds of examples, this would include the association identified between tobacco cigarette smoking and lung cancer, supine rather than prone infant sleep position to prevent sudden infant death syndrome, and folic acid supplementation during pregnancy to prevent neural tube defects. In the case of the P479L variant, our studies in Alaska ${ }^{4,5}$ and those of our colleagues from Canada ${ }^{2,3}$ have consistently shown an association between homozygosity for the variant and infant death, particularly in association with infectious diseases.

In addition to questioning the significance of published epidemiologic data, the authors argue that there is little evidence of significant physiologic impact of the variant on the function of the CPT1A enzyme. However, the published evidence they cite in support of this conclusion has been both misstated and misinterpreted. For example, they incorrectly conclude that because the $\mathrm{P} 479 \mathrm{~L}$ variant reduces protein stability but does not affect the substrate binding site, it should retain catalytic activity. But in fact, loss of function due to effects on protein folding and/or stability are the most common mechanisms of pathogenicity associated with mutations causing inherited metabolic disorders, whereas mutations in substrate binding domains and catalytic sites account for only a small minority of disease-causing mutations. The authors also cite several references indicating the P479L variant form of CPT1A retains high residual enzyme activity (up to 50\%). ${ }^{6-8}$ Two of these references lacked any studies of CPT1A activity, and the high activity reported in the third reference was based on overexpression in SV40 transformed COS cells. ${ }^{7}$ In contrast they observed only $22 \%$ of wild-type activity in patient fibroblasts. They reported similar discrepancies between activities in COS cells and patient fibroblasts with several other mutant alleles of CPT1A known to be associated with the severe form of CPT1A deficiency, which they concluded was a reflection of the conditions of their in vitro system. We observed residual activity of 19\% (range 10-25) in fibroblasts from six Alaska Native infants (unpublished data), whereas much lower activity was reported in fibroblasts from Canadian Inuit and First Nations children (mean $=6 \%$, range $=2-16 \%$ ) (13).

In addition to in vitro studies demonstrating that the $\mathrm{P} 479 \mathrm{~L}$ variant has a significant impact on CPT1A activity, fasting studies have shown that Alaska Native children homozygous for the variant have a significant impairment in the ability to generate ketones during fasting. ${ }^{9}$ However, the authors misinterpret the observation that only two of the five children in our study became hypoglycemic as suggesting that homozygosity for the variant is not associated with an increased risk of hypoglycemia. This study was designed to allow for an assessment of the physiologic response to fasting, and at the same time to try and avoid hypoglycemia, which is a relatively late consequence of fasting in disorders of fatty acid oxidation. Our conclusions were based on measurement of the ratio of serum free fatty acids to ketones, which can be assessed during the transition from the use of glucose to ketones as the primary fuel, a time in the fast when the risk for hypoketotic hypoglycemia is still relatively low. ${ }^{10}$ At the end of the fast all of the children in our study had markedly elevated free fatty acid/ketone ratios (mean $=8.45 \pm 4.5$, range $4.1-16$, normal $<2.2$ ), which is highly characteristic of patients with fatty acid oxidation disorders, including severe forms of CPT1A deficiency. This is strong evidence that homozygosity for the P479L variant has significant physiologic impact, and also indicates a very high likelihood that all of the children would have become hypoglycemic had the fast been extended. The predicted impact of this variant on fasting tolerance is borne out by the high rates of symptoms such as lethargy and hypoglycemia observed by pediatricians and other providers at the Alaska Native Medical Center, and in newborn nurseries, clinics, and hospitals throughout Alaska where children homozygous for the variant receive care. There is also anecdotal evidence from adult providers who care for elderly Alaska Native people of episodes of profound 
hypoglycemia in the elder population if they undergo a prolonged fasting event, often requiring hospitalization. Significantly, all of the children we studied were healthy at the time of the fast, but like children with other types of fatty acid oxidation disorders, they are predicted to be at increased risk of symptoms during periods of viral or bacterial illness. Consequently, we strongly disagree with the author's statement that "Further studies are needed to determine whether infectious disease aggravates any hypoglycemic risk associated with the P479L variant."

One of the public health efforts undertaken in Alaska that the authors have questioned was production of an educational video to inform parents and others about prevention of risks associated with prolonged fasting among homozygous infants. ${ }^{11}$ It includes guidelines regarding how long infants and children can safely go between meals, as well as signs and symptoms that suggest a child may need to be seen by a health-care provider. The authors state concern regarding the value of efforts "to promote dietary interventions" and "change infant feeding practices" because they are "unproven." However, the video does not include any recommendations regarding the types of foods that infants and children should eat when healthy. Additionally, the guidelines regarding fasting and interventions during illness are the same as those given to parents of all children with fatty acid oxidation disorders, which we think is merited based upon the evidence of risk for these children. The authors also state concern that "Public health messages regarding an increased risk of infant mortality could also lead to parental worry and frequent visits to health-care providers." Although the video includes no mention of infant mortality, we agree that informing parents about potential risks could lead to worry and increased health-care utilization. This issue, however, is no different for the P479L variant than for other conditions that place children at risk. And unlike many conditions, parents can effectively reduce any risk through relatively simple interventions. We consider it an obligation for health-care providers and public health agencies to inform parents about potential risks and empower them to prevent serious consequences, rather than adopting a paternalistic attitude of unilaterally deciding to withhold information until more definitive studies can be performed. We also note that this video was produced as a collaborative effort between the Division of Public Health in the Alaska Department of Health and Social Services, and Southcentral Foundation, an Alaska Native-owned, nonprofit health-care organization that serves approximately 65,000 Alaska Native and American Indian people living in the Anchorage region. Financial support for the DVD was provided by the Western States Regional Genetics Collaborative, with additional support from the Norton Sound Health Corporation, an Alaska Native-owned corporation that provides health care to Alaska Native people living in Nome and rural villages within the Norton Sound Region. Leadership from both Southcentral Foundation and Norton Sound Health Corporation, along with leadership from many other Tribal Health organizations, was in full support of the educational DVD to provide information on the P479L variant for families. Further evidence of Tribal Health support for education about the P479L variant is demonstrated by the efforts undertaken by the Department of Pediatrics at the Alaska Native Medical Center to educate and provide medical support to all health-care providers in Alaska who care for children with the P479L variant. This includes incorporation of information about fasting and other concerns in homozygous children into a handbook written for health-care providers working with Alaska Native patients. ${ }^{12}$

Another public health effort that the authors have questioned is the identification of infants with two copies of the variant by newborn screening. As stated above, we think that parents have a right to know about potential risks to their children's health, especially when simple interventions can significantly reduce that risk. The P479L variant was first identified in Alaska after the initiation of expanded newborn screening by tandem mass spectrometry. However, we subsequently found that tandem mass spectrometry-based screening based on standard algorithms used for other US populations has extremely low sensitivity for the P479L variant, and that changes in the screening algorithm to increase sensitivity would reduce the specificity and positive predictive value below levels acceptable for population-based screening. ${ }^{13}$ Consequently, in July 2016 the State of Alaska added universal DNA testing for the P479L variant to its newborn screening panel, allowing for the identification of all homozygous infants.

Finally, the authors have called for more research, not just regarding health effects of the P479L variant, but also evaluations of other known and suspected risk factors. We agree with this assessment. Consistent with this, we have included other known risk factors for adverse childhood health in all of our prior analyses, and the studies have been run by a team with broad experience in child health, infant mortality, and newborn screening, rather than persons interested only in a specific genetic variant. More recently, we have begun a collaborative research effort with a team that includes Alaska Native investigators to assess the potential role of polyunsaturated fats contained in traditional Alaska Native foods as modifiers of the health risks associated with the variant. As in previous studies we will include information about other potential risk factors in our analysis. The initiation of universal DNA testing for the P479L variant will also help expand knowledge regarding health effects of this variant. The authors have suggested other research priorities that include topics not related to fatty acid oxidation or newborn screening. Clearly, indigenous Arctic populations, as with all populations, have many health priorities. However, given all of the evidence of health risks associated with the P479L variant, and considering that a definitive diagnostic test exists, the severity of the outcomes in question (hypoglycemia, death, and hospitalization), and the availability of a simple and effective intervention, we think it would be irresponsible to delay action for years while awaiting the results of more 
definitive causal assessments and research into other risk factors.

\section{DISCLOSURE}

The authors declare no conflict of interest.

David M. Koeller, $M D^{1}$, Matt Hirschfeld, $M D, P h D^{2}$, Stephanie Birch, MPH, FNP ${ }^{3}$, Thalia Wood, MPH ${ }^{4,8}$, Rebekah Morisse, BSN, MPH', Sabra Anckner, RN, BSN ${ }^{6}$ and Bradford D. Gessner, MD, MPH ${ }^{7,9}$

${ }^{1}$ Departments of Molecular \& Medical Genetics and Pediatrics, Oregon Health \& Science University, Portland, Oregon, USA; ${ }^{2}$ Maternal Child Health Services, Alaska Native Medical Center, Anchorage, Alaska, USA; ${ }^{3}$ Women's, Children's and Family Health, Alaska Division of Public Health, Anchorage, Alaska, USA; ${ }^{4}$ Children's Health Unit, Section of Women's Children's and Family Health, Alaska Division of Public Health, Anchorage, Alaska, USA; ${ }^{5}$ Perinatal \& Early Childhood Health Unit, Section of Women's Children's and Family Health, Alaska Division of Public Health, Anchorage, Alaska, USA; ${ }^{6}$ Section of Women's, Children's and Family Health, Alaska Division of Public Health, Anchorage, Alaska, USA; ${ }^{7} \mathrm{MCH}$ Epidemiology Unit, Section of Women's, Children's, and Family Health, Alaska Division of Public Health, Anchorage, Alaska, USA. Correspondence: David M. Koeller (koellerd@ohsu.edu)

${ }^{8}$ Retired.

${ }^{9}$ Current affiliation: EpiVac Consulting, Anchorage, Alaska, USA.

\section{REFERENCES}

1. Fohner AE, Garrison NA, Austin MA, Burke W. Carnitine palmitoyltransferase 1A P479L and infant death: policy implications of emerging data. Genet Med; e-pub ahead of print 26 January, 2017.

2. Collins SA, Surmala P, Osborne $G$, et al. Causes and risk factors for infant mortality in Nunavut, Canada 1999-2011. BMC Pediatr. 2012;12:190.

3. Sinclair GB, Collins S, Popescu O, McFadden D, Arbour L, Vallance HD. Carnitine palmitoyltransferase I and sudden unexpected infant death in British Columbia First Nations. Pediatrics. 2012;130:e1162-1169.
4. Gessner BD, Gillingham MB, Birch S, Wood T, Koeller DM. Evidence for an association between infant mortality and a carnitine palmitoyltransferase 1A genetic variant. Pediatrics. 2010;126:945-951.

5. Gessner BD, Wood T, Johnson MA, Richards CS, Koeller DM. Evidence for an association between infant mortality and homozygosity for the arctic variant of carnitine palmitoyltransferase 1A. Genet Med. 2016;18: 933-939.

6. Bonnefont JP, Djouadi F, Prip-Buus C, Gobin S, Munnich A, Bastin J. Carnitine palmitoyltransferases 1 and 2: biochemical, molecular and medical aspects. Mol Aspects Med. 2004;25:495-520.

7. Brown NF, Mullur RS, Subramanian I, et al. Molecular characterization of L-CPT I deficiency in six patients: insights into function of the native enzyme. J Lipid Res. 2001;42:1134-1142.

8. Rajakumar C, Ban MR, Cao H, Young TK, Bjerregaard P, Hegele RA. Carnitine palmitoyltransferase IA polymorphism P479L is common in Greenland Inuit and is associated with elevated plasma apolipoprotein A-I. J Lipid Res. 2009;50:1223-1228.

9. Gillingham MB, Hirschfeld $M$, Lowe $S$, et al. Impaired fasting tolerance among Alaska Native children with a common carnitine palmitoyltransferase 1A sequence variant. Mol Genet Metab. 2011;104:261-264.

10. Bonnefont JP, Specola NB, Vassault $A$, et al. The fasting test in paediatrics: application to the diagnosis of pathological hypo- and hyperketotic states. Eur J Pediatr. 1990;150:80-85.

11. Copyright (C) 2013 Southcentral Foundation. The Other Energy Crisis: Arctic Variant CPT1A. https://www.youtube.com/watch?v=g-JRZ7PO3yk\& feature =youtu.be. Published on June 25, 2013.

12. Jardine B. Pocket Guide to Alaska Native Pediatric Diagnoses: Review of Diagnoses Rarely Seen in Other Populations. Alaska Native Tribal Health Consortium: Anchorage, AK, 2016.

13. Gessner $B D$, Gillingham MB, Johnson MA, et al. Prevalence and distribution of the $\mathrm{C} .1436 \mathrm{C} \rightarrow \mathrm{T}$ sequence variant of carnitine palmitoyltransferase 1A among Alaska Native infants. J Pediatr. 2011;158: 124-129.

Advance online publication 3 August 2017. doi:10.1038/gim.2017.116 